\title{
Talar Dome Investigation and Talocrural Joint Axis Analysis Based on Three-Dimensional (3D) Models: Implications for Prosthetic Design
}

\author{
Da-Hang Zhao $\mathbb{D}^{1},{ }^{1,2}$ Di-Chao Huang $\left(\mathbb{D},{ }^{1,3}\right.$ Gong-Hao Zhang, ${ }^{1}$ Yun-Ping Fan $\left(\mathbb{D},{ }^{4}\right.$ Jian Yu $\left(\mathbb{D},{ }^{1}\right.$ \\ Shao-Bai Wang $\mathbb{D}^{4,5}$ Kan Wang $\mathbb{D}^{6}$, and Xin Ma $\mathbb{D}^{1}$ \\ ${ }^{1}$ Department of Orthopedics, Huashan Hospital, Fudan University, Shanghai, China \\ ${ }^{2}$ Department of Orthopaedics, Ruijin Hospital, Shanghai Jiaotong University School of Medicine, Shanghai, China \\ ${ }^{3}$ Department of Traumatic Orthopaedics, Ningbo No. 6 Hospital, Zhengjiang, China \\ ${ }^{4}$ Shanghai InnoMotion Inc., Shanghai, China \\ ${ }^{5}$ Key Laboratory of Exercise and Health Science of Ministry of Education, Shanghai University of Sport, Shanghai, China \\ ${ }^{6}$ Department of Radiology, Huashan Hospital, Fudan University, Shanghai, China
}

Correspondence should be addressed to Xin Ma; maxinhuashan@163.com

Received 18 April 2019; Revised 3 August 2019; Accepted 5 September 2019; Published 7 November 2019

Academic Editor: Friedrich P. Paulsen

Copyright ( $\odot 2019$ Da-Hang Zhao et al. This is an open access article distributed under the Creative Commons Attribution License, which permits unrestricted use, distribution, and reproduction in any medium, provided the original work is properly cited.

\begin{abstract}
Ankle joint kinematics is mainly stabilized by the morphology of the talar dome and the articular surface of tibiofibular mortise as well as the medial and lateral ligament complexes. Because of this the bicondylar geometry of talus dome is believed to be crucial for ankle implant design. However, little data exist describing the precise anatomy of the talar dome and the talocrural joint axis. The aim of this study is to document the anatomy of the talar dome and the axis of the talocrural joint using three-dimensional (3D) computed tomographic (CT) modeling. Seventy-one participants enrolled for CT scanning and 3D talar model reconstruction. All the ankles were held in a neutral position during the CT scanning. Six points on the lateral and medial crest of the talar dome were defined. The coordinate of the six points; radii of lateral-anterior (R-LA), lateral-posterior (R-LP), medialanterior (R-MA), and medial-posterior (R-MP) sections; and inclination angle of the talar dome were measured, and the inclination and deviation angles of the talocrural joint axis were determined. The mean values of R-LA, R-LP, R-MA, and R-MP were $19.23 \pm 2.47 \mathrm{~mm}, 18.76 \pm 2.90 \mathrm{~mm}, 17.02 \pm 3.49 \mathrm{~mm}$, and $22.75 \pm 3.04 \mathrm{~mm}$. The mean inclination angle of the talar dome was $9.86 \pm 3.30$ degrees. Gender variation was found in this parameter. The mean inclination and deviation angles were $8.60 \pm 0.07$ and $0.76 \pm 0.69$ degrees for the dorsiflexion axis and $-7.34 \pm 0.07$ and $0.09 \pm 0.18$ degrees for the plantarflexion axis. Bilateral asymmetries between the medial and lateral crest of the talar dome were found, which resulted in different dorsiflexion and plantarflexion axes of the talocrural joint. Currently, no ankle implants replicate this talar anatomy, and these findings should be considered in future implant designs.
\end{abstract}

\section{Introduction}

Quantitative investigations of the complex morphology of the talar dome, which articulates with the tibial plafond and mainly conducts coupled motion with the rotational component of dorsiflexion and plantarflexion, are crucial for understanding the joint function and designing ankle implants [1-5]. The main articular surface of the current ankle implant is between talar component and polyethylene (PE) insert. The design features of current ankle implant are quite different, and there exist some failures of total ankle arthroplasty (TAA) resulted from poor prosthesis designs [6-8].

It was controversial on the radius of talar dome morphology and the direction of the talocrural joint axis from the published studies. Although superior surface of the talar dome was typically pulley shape with a central mild concaved surface and two asymmetrical borders [9], 
early studies suggested that the trochlear surface could be regarded as a frustum, in which the apex directed medially and the rotation axis coincided with the line connecting the medial and lateral malleolus tips. Furthermore, the medial border of the talar dome had a smaller radius of curvature than that of lateral border $[10,11]$. And these observations have been copied in the design of some ankle systems [12-14]. However, the concept of the fixed rotation axis of the talocrural joint contradicted with the coupled behavior of ankle joint complex, and several later results in vitro or vivo $[4,15-18]$. Some recent research studies revealed that the talar dome was a saddle-shaped, skewed, truncated cone with laterally oriented apex and the radius of curvature of the lateral border was smaller than the medial border without the fixed rotation axis [19-21], which were different from previous conclusions [10]. Furthermore, the unilateral and bilateral asymmetries between the radii of the medial and lateral talar trochleae were found by dividing the trochleae into anterior and posterior regions, in which the anteromedial radius was smaller than anterolateral or posteromedial radius [22]. These results were similar to one early study that medial trochlea had two parts of different radii, whereas the lateral trochlea had one radius [23].

Certain amount of error might arise by current methods for talar measurement. On the one hand, intrinsic error could exist when measuring cadaver specimens or manually determining subject points on the three-dimensional (3D) reconstructed model. Thus, the coordinate system established by the landmarks might not be accurate $[10,11,22-25]$. On the other hand, not many studies positioned the foot and lower extremity in the neutral position, in which both the longitudinal axis of the second metatarsal and mechanical axis were parallel to the sagittal plane of gantry during CT image collection [26].

The purpose of this study was to investigate (1) the relative distribution of circles on the lateral and medial crests of the talar dome, (2) the radii of circles, and (3) the talocrural joint axis based on 3D reconstructed model scaling and alignment.

\section{Materials and Methods}

2.1. Study Participants. Eighty-four healthy participants registered initially. Medical history was recorded to exclude the participants with previous trauma. Two of the authors (DZ and GZ) evaluated the bilateral ankle X-ray independently to exclude the participants with deformity or degenerative changes. The protocol was approved by the Ethics Committee of Huashan Hospital, Fudan University. Seventy-one participants enrolled in this study including 56 males and 15 females. The mean age was 25.17 (21 to 37). The mean height, weight, and body mass index (BMI) were $173.72 \pm 6.22 \mathrm{~cm}, 68.48 \pm 11.16 \mathrm{~kg}$, and $22.62 \pm 2.94$. A male participant, whose height $(172 \mathrm{~cm})$ and weight $(67 \mathrm{~kg})$ were most close to the mean value, was selected as the reference participant. Finally, one reference and 70 target participants were included.
2.2. Reconstruction of the $3 D$ Models. Computed tomography (CT) images of all the participants were taken from $10 \mathrm{~cm}$ proximal to the tibiotalar joint to the sole in our institution (Brilliance iCT, Philips, Cleveland, Ohio, USA) using axial slices $(120 \mathrm{kV}, 250 \mathrm{~mA}$, slice thickness $=0.67 \mathrm{~mm}$, slice increment $=0.67 \mathrm{~mm}$, and pixel size $=512 \times 512$ matrix). Digital Imaging and Communications in Medicine (DICOM) images were imported into Mimics (Version 9.0, Materialise NV, Belgium). After proper segmentation, filtering, and rendering, 3D talar models were obtained with stereo lithography (STL) format. All the models were then imported into Geomagic Studio 2013 (Geomagic, Morrisville, North Carolina, USA) to refine the geometry. As left and right tali show a strong degree of symmetry [27], we randomly selected one side for CT scanning. Finally 34 right and 37 left feet and ankles, of which right side was selected for the reference participant, were included.

\subsection{Position of the Reference Participant during CT Scanning.} Right foot and ankle CT images were collected when half weight-bearing was loaded on the reference participant using a foot-loading device, which was used in our published studies $[28,29]$. The device includes a foot plate with stress sensor, a frame, and a loading control system (Figure 1(a)), in which the foot plate is parallel to the coronal plane of CT gantry and the frame is parallel to the ground. The right foot sole of the reference participant was placed on the foot plate with left knee in flexion. The right leg was supported by a radiolucent pad to make it perpendicular to the foot plate, and then half weight was loaded on the reference participant by the loading control system (Figure 1(b)). A $5 \mathrm{~cm}$ length Kirschner wire $(2 \mathrm{~mm} \Phi)$ was marked along with the axis of the second metatarsal (Figure 1(c)). During CT image collection, the foot and ankle were positioned in the neutral position by making sure the extension line of Kirschner wire passing through the midwidth of the talar dome simultaneously coincides with the mechanical axis (Figure 1(d)).

2.4. Model Scaling, Alignment, and Coordinate System Established. First, left models were transferred to right using left-right mirror transform. Next, a uniform scaling approach was used to quantify the volume of target models to the reference model, and then target models were aligned to the reference model using built-in algorithm "best-fit alignment" [30, 31]. Finally, each target model was compared to the reference model to figure out the deviations. The volume, scaling factor, maximum, mean, and standard deviation were recorded (see Supplementary data 1 ). The mean standard deviation was $1.02 \mathrm{~mm}$. The transverse plane was parallel to the foot plate of the loading device. The coronal plane was perpendicular to the ground; the sagittal plane was defined as a plane perpendicular to both the transverse and coronal planes. The origin of the coordinate system was selected as the anterior point of lateral part of the trochlea (Figures 2(a) and 2(b)). The $X, Y$, and $Z$ axes all passed through the origin and were perpendicular to sagittal, coronal, and transverse planes, respectively (Figures 2(c)-2(e)). 


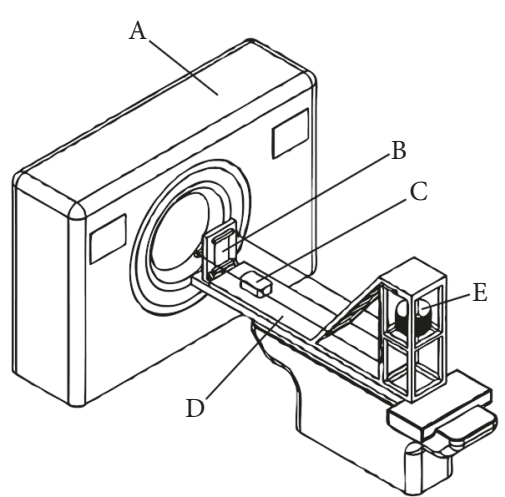

(a)

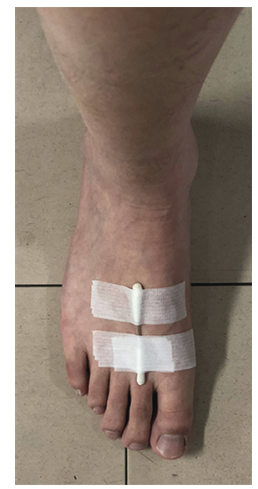

(c)

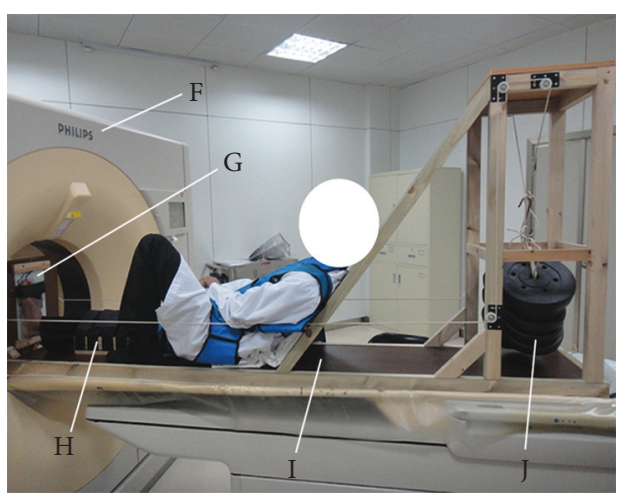

(b)

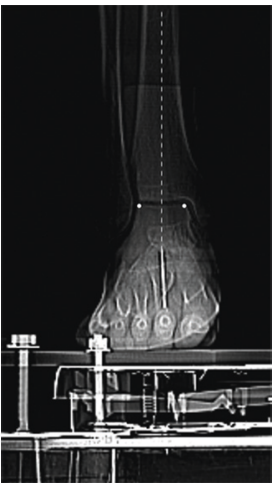

(d)

FIGURE 1: Foot-loading device was used when CT scan was performed on the reference participant. (a) The main device included a CTgantry (A), a foot plate with stress sensor (B), a radiolucent pad (C), a frame (D), and a loading control system (E). (b) The reference participant was sitting on the foot-loading device which included a CT gantry (F), a foot plate with stress sensor $(\mathrm{G})$, a radiolucent pad (H), a frame (I), and a loading control system (J). (c) A $5 \mathrm{~cm}$ Kirschner wire $(2 \mathrm{~mm} \Phi)$ was marked along with the axis of the second metatarsal by the landmark on the skin. (d) Making sure the extension line of the Kirschner wire passes through the midwidth of the talar dome and coincides with the mechanical axis during CT scanning.

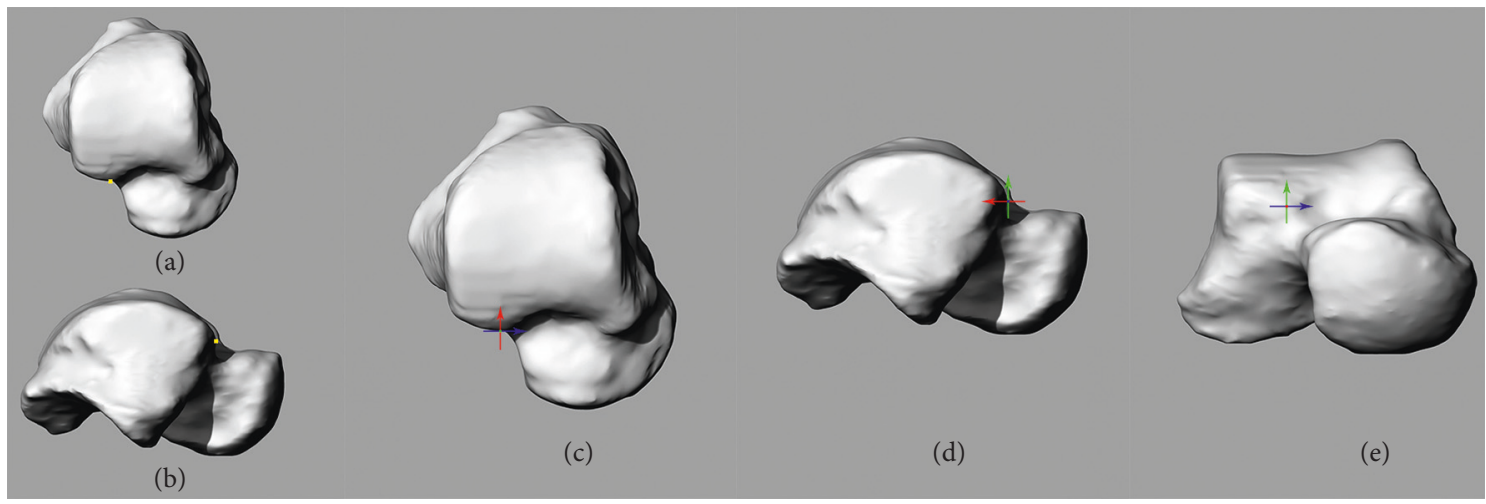

Figure 2: Coordinate system. (a) Origin of the coordinate system was observed from top view. (b) Origin was observed from lateral view. (c) $X$ and $Y$ axes were perpendicular to sagittal and coronal planes. (d) $Y$ and $Z$ axes were perpendicular to coronal and transverse planes. (e) $X$ and $Z$ axes were perpendicular to sagittal and transverse planes.

2.5. Definition of the Points for Measurement. First, lateraltop (LT) and medial-top (MT) points were defined as the vertex of the lateral and medial crests of the talar dome (Figure 3(a)). Second, two transverse outlines on the talar dome, which were $8 \mathrm{~mm}$ beneath LT and $5 \mathrm{~mm}$ beneath MT, were obtained (Figure 3(b)). Third, two tangent points, which were made by two 45-degree lines on the transverse outline $8 \mathrm{~mm}$ beneath LT, were created on the lateral side (Figure 3(c)). Fourth, the lateral crest plane of the talar dome, which passed through LT and was perpendicular to the transverse plane and parallel to the line connecting the two lateral tangent points, was created (Figure 3(c)). Fifth, 


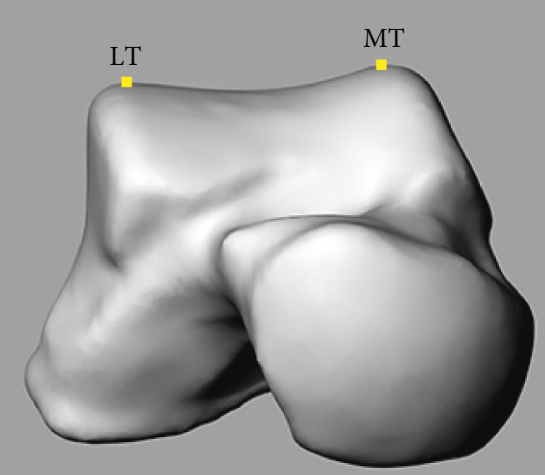

(a)

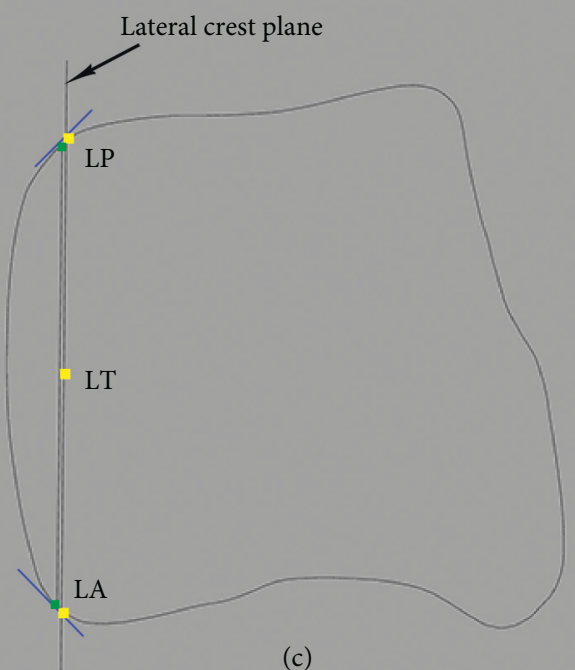

(c)

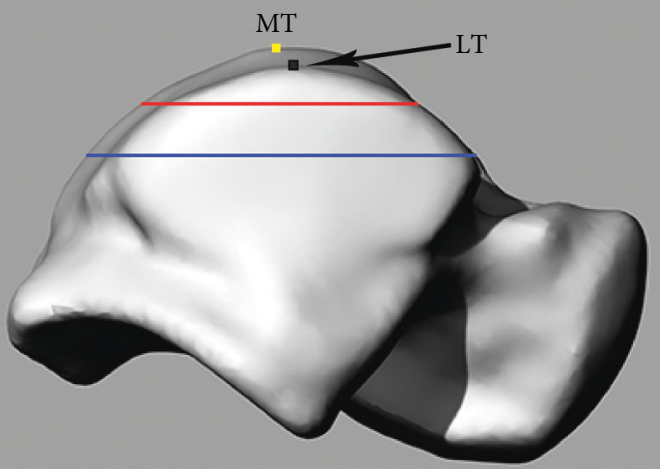

(b)

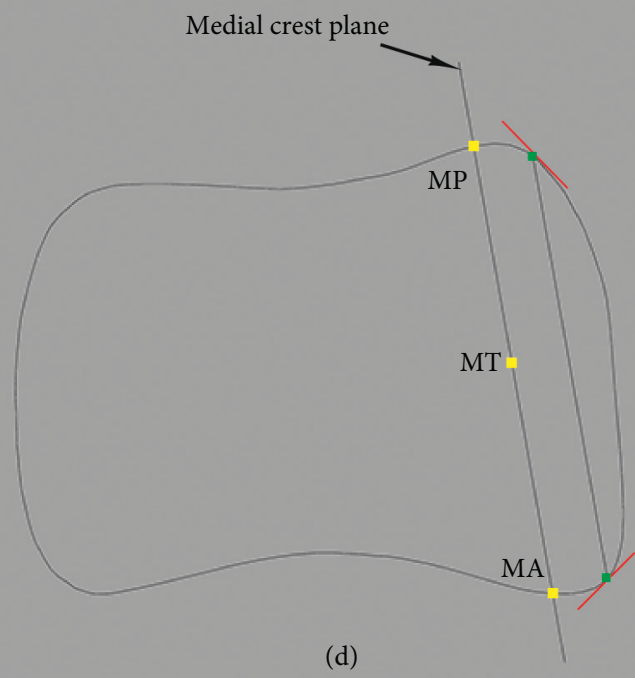

FIGURE 3: Six points on the circles of lateral and medial crests of the talar dome were defined. (a) LT and MT were defined as the vertex of the lateral and medial crests of the talar dome from front view. (b) Two transverse outlines of the talar dome were obtained including one $8 \mathrm{~mm}$ beneath LT (blue line) another $5 \mathrm{~mm}$ beneath MT (red line). (c) Two tangent points (green points) were created by two 45-degree lines (blue lines), and the lateral crest plane created two intersections (yellow points) on the transverse outline. (d) Medial crest plane created two intersections (yellow points) on the transverse outline in the same way.

lateral-anterior (LA) and lateral-posterior (LP) points, which were the two intersections between the lateral crest plane and transverse outline $8 \mathrm{~mm}$ beneath LT, were created (Figure 3(c)). Finally, medial-anterior (MA) and medialposterior (MP) points on the transverse outline $5 \mathrm{~mm}$ beneath MT were created in the same way (Figure 3(d)).

2.6. Coordinate and Reconstructed Model. Coordinates of the six points for 71 talar models were measured two times by one author (DZ) (see Supplementary data 2). Relative distribution of circles on the lateral and medial crests of talar dome included category $\mathrm{I}$, in which $Y$ coordinate and $Z$ coordinate of LT were both less than MT, category II, in which $Y$ coordinate of LT was more than MT and $Z$ coordinate of LT was less than MT, category III, in which $Y$ coordinate and $Z$ coordinate of LT were both more than MT, and category IV, in which $Y$ coordinate of LT was less than MT and $Z$ coordinate of LT was more than MT.

2.7. Radius and Inclination Angle of the Talar Dome. Two horizontal lines on lateral and medial crest planes which passed through LT and MT, respectively, were created 
(Figures 4(a) and 4(b)). A lateral-anterior circle which passed through LT and LA and tangent to the horizontal line was created on the lateral crest plane. The radius of this circle was defined as the radius of lateral-anterior (R-LA) section of the talar dome. Likewise, the circles and radii of lateralposterior (R-LP), medial-anterior (R-MA), and medialposterior (R-MP) sections were defined. Furthermore, the angle between lateral and medial crest planes was defined as inclination angle of the talar dome (Figure 4(c)). Four radii and inclination angles of each model were calculated (see Supplementary data 3).

2.8. Talocrural Joint Axis and the Orientation. A half truncated cone model based on the mean coordinates was reconstructed. And vertical distance from LT point to the bottom transverse plane was $10 \mathrm{~mm}$ (Figure 5) .This reconstructed model was used for talocrural joint axis analysis. The dorsiflexion axis of the talocrural joint was defined as a line passing through the centers of the lateralanterior and medial-anterior circles. The plantarflexion axis was defined as a line passing through the centers of the lateral-posterior and medial-posterior circles. The inclination angle was defined as the angle of the talocrural joint axis in the coronal plane, in which a positive value indicated superior oriented axis in the medial direction. The deviation angle was defined as the angle in the transverse plane, in which a positive value indicated anterior oriented axis in the medial direction. The inclination and deviation angles were measured when the reconstructed model was rotated from the neutral position to 30 degrees anterior and 30 degrees posterior with 5 degrees interval. When rotating the model, make sure two points, which included the apexes on the lateral and medial crests of the rotated model, are on the line which connected the mean LT and mean MT point.

2.9. Statistical Analysis. A two-tailed unpaired $t$-test was used to investigate gender variations in the inclination angle of the talar dome. A single-factor ANOVA was utilized to identify the differences between R-LA, R-LP, R-MA, and R-MP. When a difference was found, least significant difference (LSD) pairwise multiple comparison tests were applied. SAS software version 9.2 (SAS Institutes, Cary, North Carolina, USA) was used, and a $p$ value of $<0.05$ was considered significant.

\section{Results}

3.1. Relative Distribution of Lateral and Medial Circles. According to the relative distribution category, thirty models belonged to category I, 36 category II, 2 category III, and 3 category IV (Table 1). Mean coordinate of LT, MT, LA, LP, $\mathrm{MA}$, and MP indicated the relative distribution of the lateral and medial circles belonged to category II (Table 2). The reconstructed half truncated cone model, which represented the mean value of the 71 talar models and consisted of two parts of skewed, truncated cones with different oriented apexes, could be considered as the section between the lateral and medial crest planes (Figure 5).
3.2. Radius and Inclination Angle of the Talar Dome. The mean values of R-LA, R-LP, R-MA, and R-MP were $19.23 \pm 2.47 \mathrm{~mm}, \quad 18.76 \pm 2.90 \mathrm{~mm}, \quad 17.02 \pm 3.49 \mathrm{~mm}$, and $22.75 \pm 3.04 \mathrm{~mm}$ (Table 3). Significant statistical differences were found between most of the multiple comparisons including R-LA and R-MA, R-LP and R-MP, and R-MA and R-MP $(p<0.001)$; however, no statistical difference ( $p=0.356$ ) was found between R-LA and R-LP (Figure 6). The mean inclination angle of the talar dome was $9.86 \pm 3.30$ degrees, which was $8.79 \pm 2.33$ degrees for males and $13.87 \pm 3.38$ degrees for females, respectively. Gender variation was found in this parameter $(p<0.001)$.

3.3. Inclination and Deviation Angles of the Talocrural Joint Axis. The inclination and deviation angles constantly and slightly changed along with the reconstructed model when the reconstructed half truncated cone model was rotated from the neutral position to 30 degrees anterior and 30 degrees posterior (Figure 7). The direction of inclination angle was mainly fixed; however, the direction of the deviation angle was changed during dorsiflexion or plantarflexion, especially for the deviation angle of the plantarflexion axis. The mean inclination and deviation angles were $8.60 \pm 0.07$ and $0.76 \pm 0.69$ degrees for the dorsiflexion axis and $-7.34 \pm 0.07$ and $0.09 \pm 0.18$ degrees for the plantarflexion axis (Table 4).

\section{Discussion}

Better restoration of the morphology of the talar dome is essential to improve the biomechanical research and ankle implant design [11]. However, the results on the radius of the talar dome were controversial, and the repeatability of their methods was not so good [19-22, 24, 26]. The current study found that bilateral asymmetry circles of the medial and lateral crests of the talar dome with four parts of different radii resulted in dorsiflexion and plantarflexion axes constantly changing throughout the talocrural joint motion.

Some limitations existed. First, the neutral position for the foot, ankle, and lower extremity of reference participant was set by the landmark on the skin, which might result in some errors. With the radiographic assessment, it was thought the foot and ankle of the reference participant could be set in neutral position and aligned with ipsilateral lower extremity during CT image collection. Second, rotating the model in this study could not simulate the real talocrural joint motion because the talar simultaneously rotates and slides in the ankle mortise during dorsiflexion and plantarflexion, which were determined and stabilized by the morphology of talar dome and tibial platfond as well as surrounding ligament complexes $[16,32]$. It was thought that the simplified motion could not affect the main direction of the talocrural joint axis.

Although 4 categories of the LT and MT relative distribution existed, MT of most models was higher than LT (66/71). The mean $Y$ coordinate of LT and MT was nearly the 


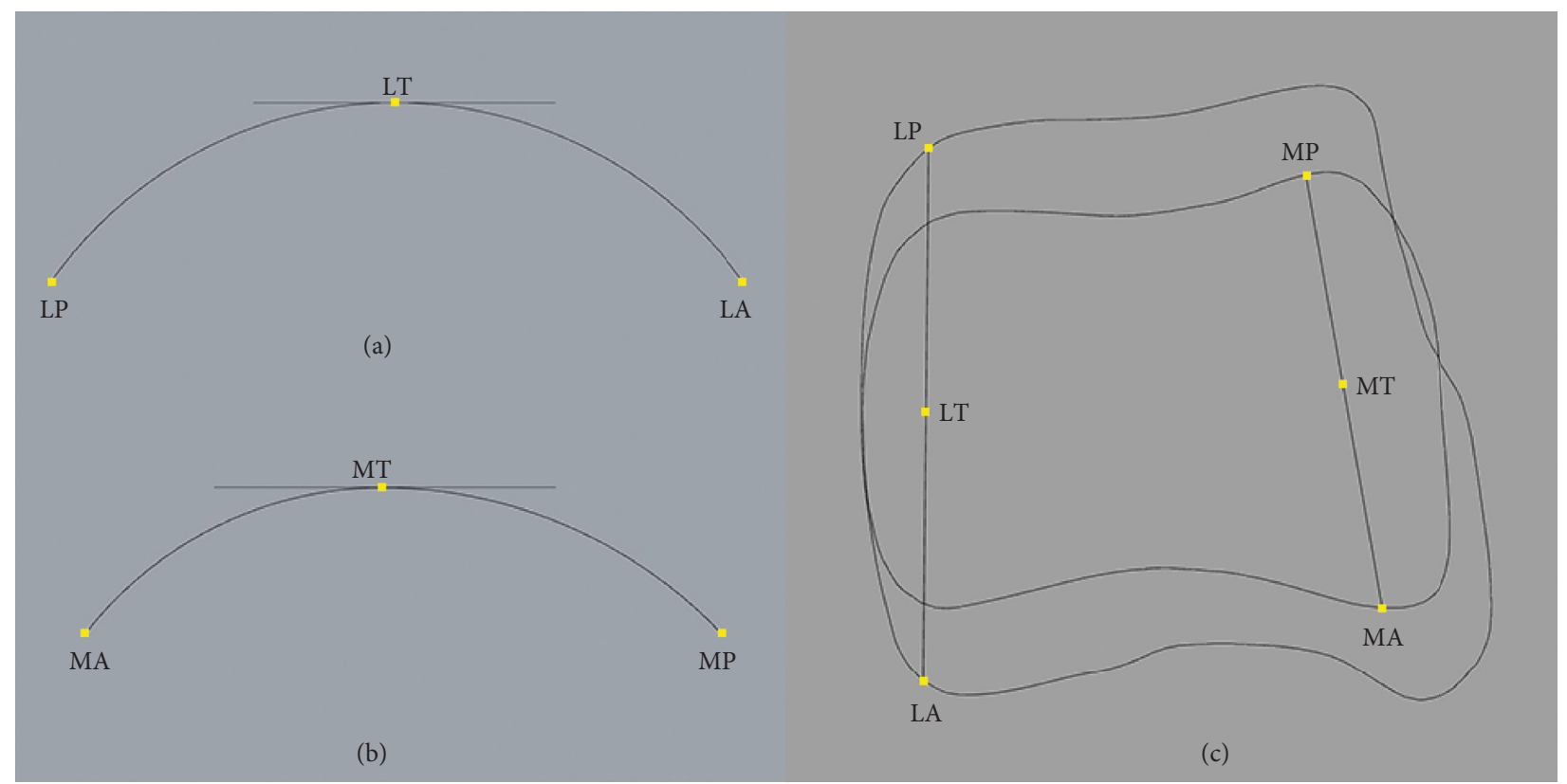

FIgURE 4: Four parts of circles were defined. (a) A horizontal line which passed through LT was created on the lateral crest plane. Lateralanterior circle passed through LT and LA and was tangent to the horizontal line. Lateral-posterior circle passed through LT and LP and was tangent to the horizontal line. (b) A horizontal line which passed through MT created the medial crest plane. Medial-anterior circle passed through MT and MA and was tangent to the horizontal line. Medial-posterior circle passed through MT and MP and was tangent to the horizontal line. (c) The inclination angle of the talar dome was the angle between the plane which passed through LT, LA, and LP (lateral crest plane) and the plane which passed through MT, MA, and MP (medial crest plane).

TABLE 1: Relative distribution of lateral and medial circles.

\begin{tabular}{lccc}
\hline & $Y$ coordinate & $Z$ coordinate & Number of models \\
\hline Category I & LT $>$ MT & LT $<$ MT & 30 \\
Category II & LT $<$ MT & LT $<$ MT & 36 \\
Category III & LT $<$ MT & LT $>$ MT & 2 \\
Category IV & LT $>$ MT & LT $>$ MT & 3 \\
\hline
\end{tabular}

LT: lateral-top point; MT: medial-top point.

TABLE 2: Mean coordinate of LT, MT, LA, LP, MA, and MP.

\begin{tabular}{lccc}
\hline & $\begin{array}{c}X \text { coordinate } \\
(\mathrm{mm})\end{array}$ & $\begin{array}{c}Y \text { coordinate } \\
(\mathrm{mm})\end{array}$ & $\begin{array}{c}Z \text { coordinate } \\
(\mathrm{mm})\end{array}$ \\
\hline LT & -5.23 & 16.94 & 9.89 \\
LA & -5.83 & 1.40 & 1.89 \\
LP & -4.65 & 32.21 & 1.89 \\
MT & 17.59 & 16.88 & 10.98 \\
MA & 19.18 & 5.03 & 5.98 \\
MP & 15.70 & 30.93 & 5.98 \\
\hline
\end{tabular}

LT: lateral-top point; MT: medial-top point; LA: lateral-anterior point; LP: lateral-posterior point; MA: medial-anterior point; MP: medial-posterior point.

same (mean $Y$ coordinate of MT was $0.06 \mathrm{~mm}$ less than LT), instead the lateral circle was about $1 \mathrm{~mm}$ below the medial circle. This indicated the talar dome was saddle-shaped skewed truncated cone with laterally oriented apex which should be copied in ankle prosthetic design. However, a published study indicated the lateral vertex was slightly higher than the medial vertex [26]. The reason for the contradictory findings might be a result of different materials and methods used in our research. They fixed cadaver specimens in the neutral position by bone cement but could not conduct radiographic alignment of the foot and ankle with low extremity [26]. The stress sensor could confirm that all of the first and fifth metatarsal head and calcaneal tuberosity of the reference participant contact with the foot plate during CT scanning. Moreover, the mean standard deviation after model scaling and alignment was similar to that in former studies [30, 31].

Asymmetric talar component of current ankle systems had two different radii on bicondylar that the medial radius was smaller than the lateral one [7]. However, our results indicated that the medial talar dome had two different radii, whereas the lateral had approximately one, and the radius of the medial-posterior part was greater than the lateral radius, whereas the radius of the medial-anterior part was smaller than the lateral radius. This feature may help to improve the current implant design, which might be more anatomical. In this study, four circles were all defined by two points and a tangent line, respectively, which was different from former research studies in which circles were defined by at least three manually created points on lateral or medial crests of the talar dome $[11,22,25,26,33,34]$. It was found that circle defined by three points might result in the apex of the defined circle overtop the actual apex of the talar dome and laed the calculated radius bigger than the actual radius of the talar dome. The volume of the models measured in the study conducted by Nozaki et al. was not available, so we could not compare our results with theirs directly [22]. One study by Nozaki et al. also investigated four radii of the talar dome [22]. The R-LA, R-LP, and R-MP from our study were smaller and R-MA was bigger than those from their results. 


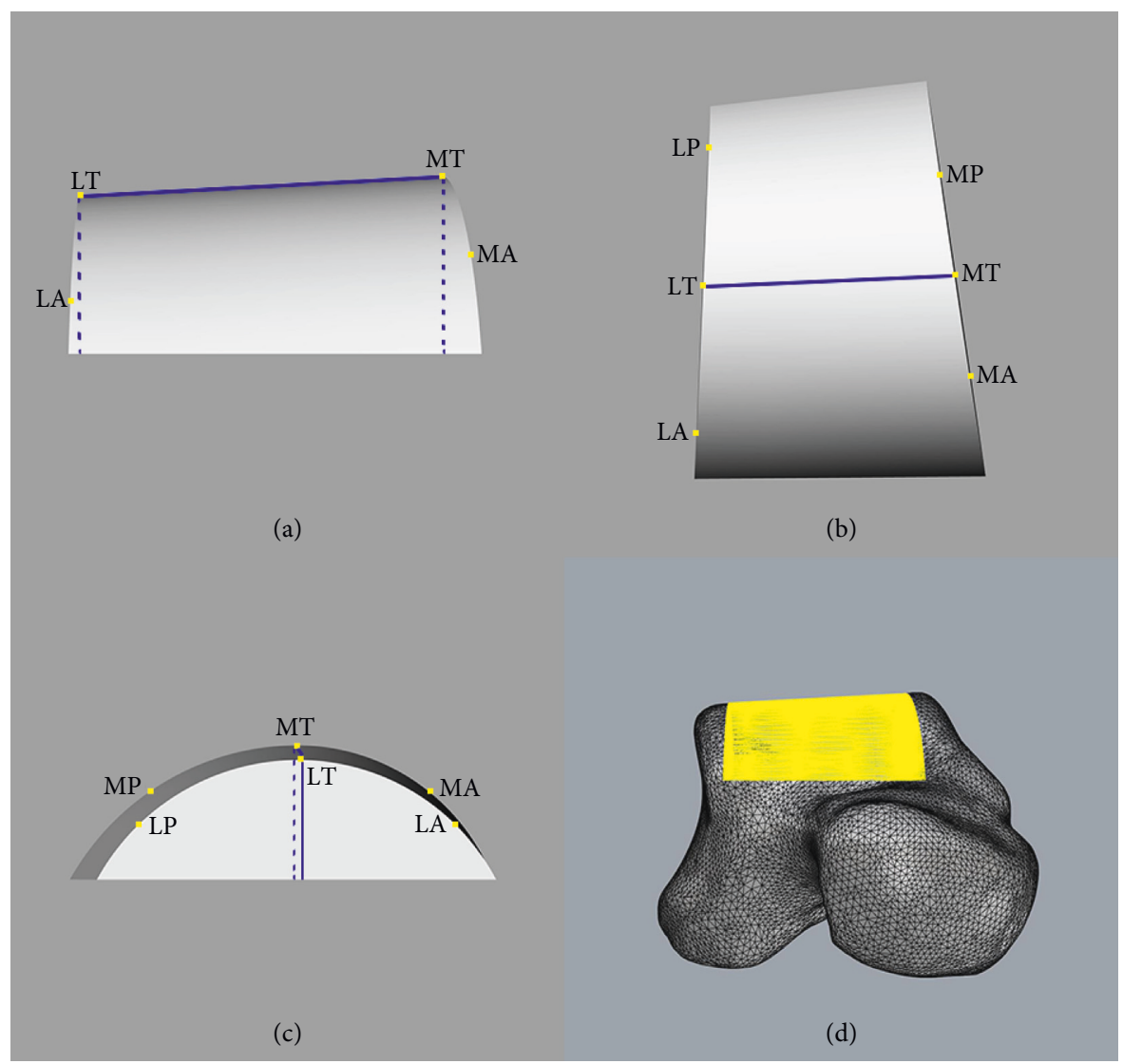

FIGURE 5: The reconstructed half truncated cone model consisted of two parts with different oriented apexes. (a) The model was observed from front view (blue solid line and dotted line divided the model into two parts). (b) The model was observed from top view (blue solid line divided the model into two parts). (c) The model was observed from lateral view (blue solid line and dotted line divided the model into two parts). (d) The model could be considered as the section between the lateral and medial crest planes of the talar dome (yellow part is the reconstructed half truncated cone model).

TABLE 3: Values of R-LA, R-LP, R-MA, and R-MP.

\begin{tabular}{lc}
\hline Variable & Value $(\mathrm{mm})$ \\
\hline R-LA & $19.23 \pm 2.47$ \\
R-LP & $18.76 \pm 2.90$ \\
R-MA & $17.02 \pm 3.49$ \\
R-MP & $22.75 \pm 3.04$ \\
\hline
\end{tabular}

Values represent means \pm standard deviation; LT: lateral-top point; MT: medial-top point; LA: lateral-anterior point; LP: lateral-posterior point; MA: medial-anterior point; MP: medial-posterior point.

This difference might result from the medial-anterior part of talus that often presents substantial osteophytes and possibly biases the true dimensions of the talar dome. However, the volume of the models measured in their study was not available, which made it impossible for us to compare the results directly. Therefore, transverse outlines $5 \mathrm{~mm}$ rather than $8 \mathrm{~mm}$ beneath MT were selected in our research. Furthermore, gender variation was found in the inclination angle of the talar dome. This proved our previous results from the $2 \mathrm{D}$ cadaveric study, and it was consistent with a $3 \mathrm{D}$ result in which the inclination angle between medial and lateral crest lines was greater in females $[26,35]$. It was believed that gender variations in the geometry existed in most bones. Similarly, the anterior-posterior to medial-

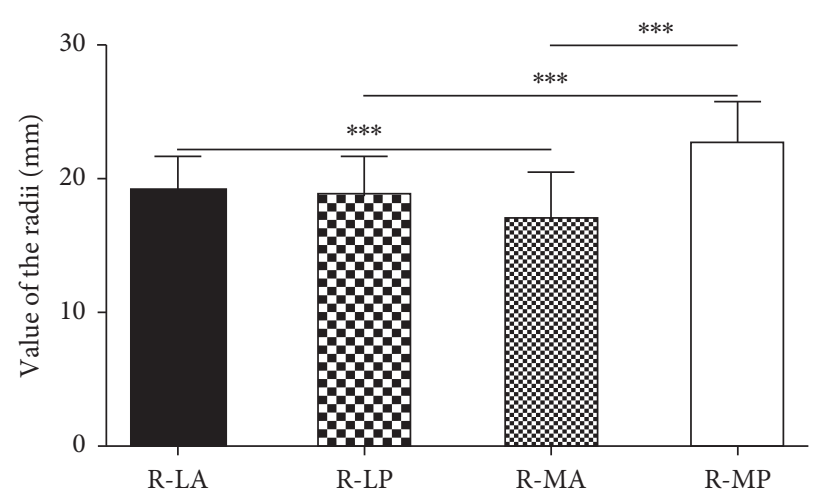

Figure 6: Comparison of the mean value of R-LA with R-LP, R-LA with R-MA, R-LP with R-MP, and R-MA with R-MP $\left({ }^{* * *} p<0.001\right)$.

lateral aspect ratio of distal femoral morphology was larger in women, which indicated that a gender-specific knee implant would reduce the potential for medial-lateral overhang [36]. Based on our results, a gender-specific shape of the talar component might be needed to reduce the potential incompatibility between the shape of the talar component and the bone cutting surface. 


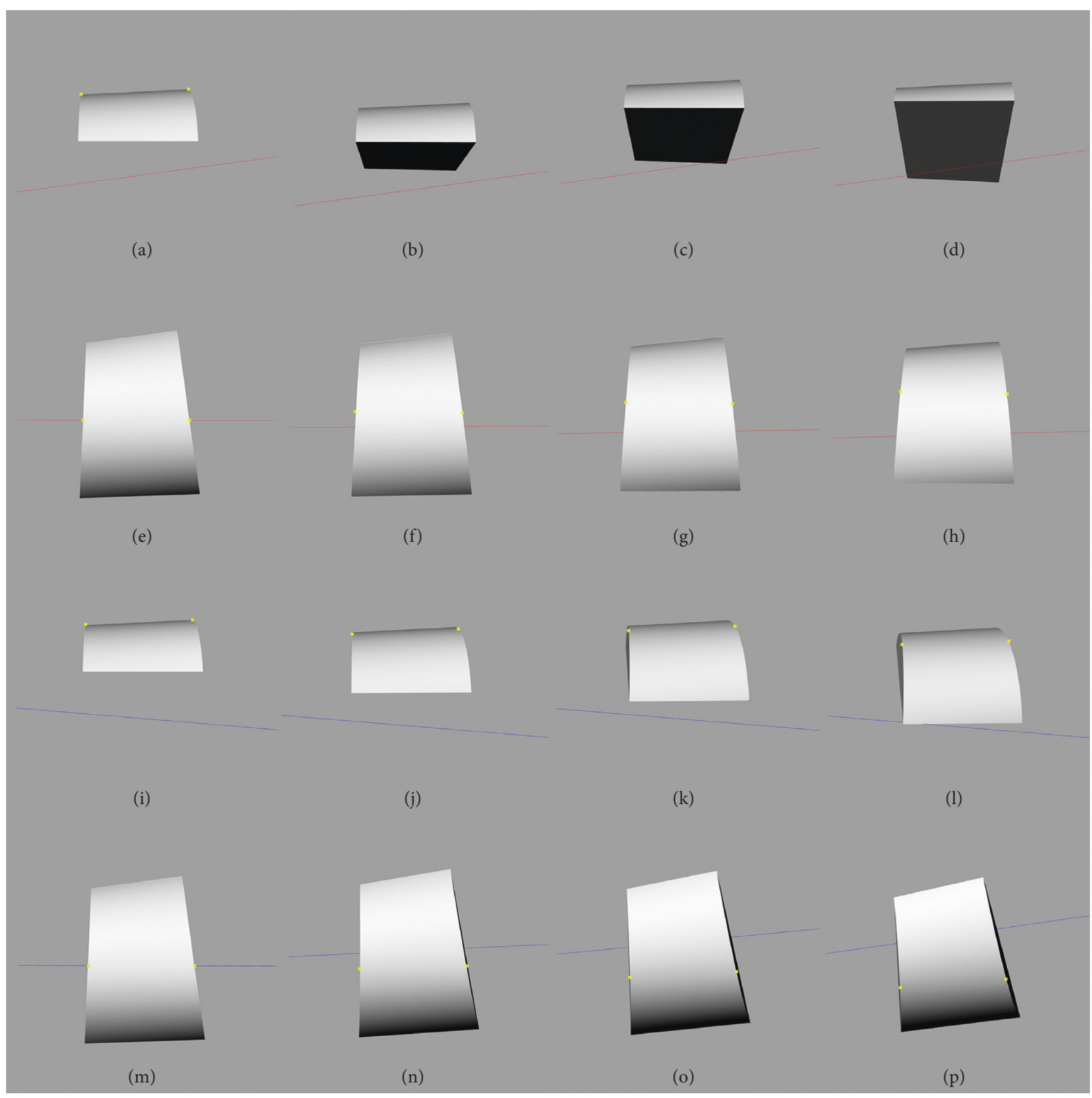

Figure 7: Talocrural joint axis analysis. (a) Inclination angles of the dorsiflexion axis at 0-degree dorsiflexion. (b) Inclination angles of the dorsiflexion axis at 10-degree dorsiflexion. (c) Inclination angles of the dorsiflexion axis at 20-degree dorsiflexion. (d) Inclination angles of the dorsiflexion axis at 30-degree dorsiflexion. (e) Deviation angles of the dorsiflexion axis at 0-degree dorsiflexion. (f) Deviation angles of the dorsiflexion axis at 10-degree dorsiflexion. (g) Deviation angles of the dorsiflexion axis at 20-degree dorsiflexion. (h) Deviation angles of the dorsiflexion axis at 30-degree dorsiflexion. (i) Inclination angles of the plantarflexion axis at 0-degree plantarflexion. (j) Inclination angles of the plantarflexion axis at 10-degree plantarflexion. (k) Inclination angles of the plantarflexion axis at 20-degree plantarflexion. (l) Inclination angles of the plantarflexion axis at 30-degree plantarflexion. (m) Deviation angles of the plantarflexion axis at 0-degree plantarflexion. (n) Deviation angles of the plantarflexion axis at 10-degree plantarflexion. (o) Deviation angles of the plantarflexion axis at 20-degree plantarflexion. (p) Deviation angles of the plantarflexion axis at 30-degree plantarflexion.

It was suggested that two different talocrural joint axes including dorsiflexion and plantarflexion existed. The reconstructed half truncated cone model consisted of two parts with different oriented apexes, which resulted from different radii of circles on the lateral and medial crests as well as their relative distribution. It was demonstrated that the axis of rotation for the talocrural joint slightly changed throughout the motion, which mainly inclined upwards medially during plantarflexion and upwards laterally during dorsiflexion, and the projections on the transverse plane were roughly parallel to the $X$ axis. These findings were consistent with the results from the published in vivo studies $[3,16]$. Consequently, the ankle implant with these asymmetric features on lateral and medial crests of the talar dome designed by us might better restore the ankle kinematics. However, the inclination and deviation angles of 
TABLE 4: Inclination and deviation angles of the dorsiflexion and plantarflexion axes.

\begin{tabular}{lcccc}
\hline $\begin{array}{l}\text { Dorsiflexion or plantarflexion } \\
\text { (degree) }\end{array}$ & $\begin{array}{c}\text { Dorsiflexion axis } \\
\text { Inclination angle } \\
\text { (degree) }\end{array}$ & $\begin{array}{c}\text { Deviation angle } \\
\text { (degree) }\end{array}$ & $\begin{array}{c}\text { Plantarflexion axis } \\
\text { Inclination angle } \\
\text { (degree) }\end{array}$ & $\begin{array}{c}\text { Deviation angle } \\
\text { (degree) }\end{array}$ \\
\hline 0 & 8.49 & -0.15 & -7.24 & -0.15 \\
5 & 8.53 & 0.13 & -7.28 & -0.08 \\
10 & 8.57 & 0.41 & -7.31 & -0.01 \\
15 & 8.61 & 0.71 & -7.34 & 0.07 \\
20 & 8.64 & 1.04 & -7.37 & 0.15 \\
25 & 8.67 & 1.39 & -7.40 & 0.25 \\
30 & 8.69 & 1.78 & -7.43 & 0.37 \\
Mean values \pm SD & $8.60 \pm 0.07$ & $0.76 \pm 0.69$ & $-7.34 \pm 0.07$ & $0.09 \pm 0.18$ \\
\hline
\end{tabular}

SD: standard deviation.

dorsiflexion and plantarflexion axes during neutral position from our research were different from the findings in a recently published study [22]. The potential reason for the variations might due to different research methods. Anterior and posterior circles of lateral or medial crests of the talar dome were tangent in our study. However, the two circles of lateral or medial crest defined in their study were separated [22].

It was found that medial crests of the talar dome consisted of two circles with different radii, of which the posterior radius was bigger, and the radius of lateral-anterior crests was nearly the same as the lateral-posterior part. The lateral radius was bigger than the medial-anterior radius but smaller than medial-posterior radius. In addition, the vertex of the medial crest was slightly higher than the lateral crest. Moreover, it is suggested these features resulted in that the dorsiflexion and plantarflexion axes were different and both slightly changed throughout the ankle motion, and the ankle kinematics might be close to physiological state. However, no talar component of the current ankle systems copied these features, which should be considered in future biomechanical and clinical research such as design of ankle implant.

\section{Data Availability}

The datasets supporting the conclusions of this article are included within the article and in the supplementary materials.

\section{Ethical Approval}

This study was conducted in accordance with the Declaration of Helsinki. The protocol was approved by the Ethics Committee of Huashan Hospital, Fudan University.

\section{Consent}

The individual consents were acquired before each donation.

\section{Conflicts of Interest}

The authors declare that they have no conflicts of interest.

\section{Authors' Contributions}

Da-Hang Zhao performed coordinates measurement, collected and analyzed the data, and wrote the manuscript. DiChao Huang performed coordinates measurement, collected the data, and recruited participants. Gong-Hao Zhang performed coordinates measurement, analyzed the data, and evaluated the X-ray. Yun-Ping Fan, Jian Yu, and Shao-Bai Wang performed volume scaling and alignment for talar models and analyzed the deviations. Kan Wang collected CT images. Xin Ma designed the study and reviewed and revised the manuscript. Da-Hang Zhao, Di-Chao Huang, and GongHao Zhang are co-first authors.

\section{Acknowledgments}

The authors would like to thank the participants who registered in this research so that the present study could be performed. This study was funded by the National Natural Science Foundation of China (grant no. 81702109), China Postdoctoral Science Foundation (grant nos. 2016M601508 and 2018T110349), and Scientific and Technological Innovation of Shanghai Science and Technology Committee (grant no. 18441902200).

\section{Supplementary Materials}

The supplementary materials of the present study included three supplementary data files, which are referred in the manuscript and are all the raw data of this original research. (Supplementary Materials)

\section{References}

[1] K. Imai, D. Tokunaga, R. Takatori et al., "In vivo three-dimensional analysis of hindfoot kinematics," Foot \& Ankle International, vol. 30, no. 11, pp. 1094-1100, 2009.

[2] S. Yamaguchi, T. Sasho, H. Kato, Y. Kuroyanagi, and S. A. Banks, "Ankle and subtalar kinematics during dorsiflexion-plantarflexion activities," Foot \& Ankle International, vol. 30, no. 4, pp. 361-366, 2009.

[3] J. M. Michael, A. Golshani, S. Gargac, and T. Goswami, "Biomechanics of the ankle joint and clinical outcomes of total ankle replacement," Journal of the Mechanical Behavior of Biomedical Materials, vol. 1, no. 4, pp. 276-294, 2008. 
[4] A. Leardini, "Geometry and mechanics of the human ankle complex and ankle prosthesis design," Clinical Biomechanics, vol. 16, no. 8, pp. 706-709, 2001.

[5] A. Leardini, J. O'Connor, F. Catani, and S. Giannini, "Mobility of the human ankle and the design of total ankle replacement," Clinical Orthopaedics and Related Research, vol. 424, no. 7, pp. 39-46, 2004.

[6] M. E. Easley, C. J. Vertullo, C. W. Urban, and J. A. Nunley, "Total ankle arthroplasty," Journal of the American Academy of Orthopaedic Surgeons, vol. 10, no. 3, pp. 157-167, 2002.

[7] C. E. Gross, A. A. Palanca, and J. K. DeOrio, "Design rationale for total ankle arthroplasty systems," Journal of the American Academy of Orthopaedic Surgeons, vol. 26, no. 10, pp. 353-359, 2018.

[8] D. Zhao, D. Huang, G. Zhang, X. Wang, T. Zhang, and X. Ma, "Positive and negative factors for the treatment outcomes following total ankle arthroplasty? A systematic review," Foot and Ankle Surgery, 2018.

[9] Kelikian and S. Armen, "Sarrafian's anatomy of the foot and ankle," Wolters Kluwer Health, Lippincott Williams \& Wilkins, Philadelphia, PA, USA, 2011.

[10] V. T. Inman, The Joints of the Ankle, Williams \& Wilkins, Baltimore, MA, USA, 1976.

[11] J. E. Bischoff, L. Schon, and C. Saltzman, "Influence of geometry and depth of resections on bone support for total ankle replacement," Foot \& Ankle International, vol. 38, no. 9, pp. 1026-1034, 2017.

[12] M. Bonnin, T. Judet, J. A. Colombier, F. Buscayret, N. Graveleau, and P. Piriou, "Midterm results of the salto total ankle prosthesis," Clinical Orthopaedics and Related Research, vol. 424, no. 3, pp. 6-18, 2004.

[13] B. Hintermann, V. Valderrabano, G. Dereymaeker, and W. Dick, "The HINTEGRA ankle: rationale and short-term results of 122 consecutive ankles," Clinical Orthopaedics and Related Research, vol. 424, no. 1, pp. 57-68, 2004.

[14] A. Barg, C. C. Bettin, A. H. Burstein, C. L. Saltzman, and J. Gililland, "Early clinical and radiographic outcomes of trabecular metal total ankle replacement using a transfibular approach," The Journal of Bone and Joint Surgery, vol. 100, no. 6, pp. 505-515, 2018.

[15] S. Siegler, J. Chen, and C. D. Schneck, "The three-dimensional kinematics and flexibility characteristics of the human ankle and subtalar joints-part I: kinematics," Journal of Biomechanical Engineering, vol. 110, no. 4, pp. 364-373, 1988.

[16] A. Lundberg, O. Svensson, G. Nemeth, and G. Selvik, "The axis of rotation of the ankle joint," The Journal of Bone and Joint Surgery. British Volume, vol. 71-B, no. 1, pp. 94-99, 1989.

[17] G. J. Sammarco, A. H. Burstein, and V. H. Frankel, "Biomechanics of the ankle: a kinematic study," Orthopedic Clinics of North America, vol. 4, no. 1, pp. 75-96, 1973.

[18] R. J. de Asla, L. Wan, H. E. Rubash, and G. Li, "Six DOF in vivo kinematics of the ankle joint complex: application of a combined dual-orthogonal fluoroscopic and magnetic resonance imaging technique," Journal of Orthopaedic Research, vol. 24, no. 5, pp. 1019-1027, 2006.

[19] S. Siegler, J. Toy, D. Seale, and D. Pedowitz, "The clinical biomechanics award 2013-presented by the international society of biomechanics: new observations on the morphology of the talar dome and its relationship to ankle kinematics," Clinical Biomechanics, vol. 29, no. 1, pp. 1-6, 2014.

[20] C. Belvedere, S. Siegler, A. Ensini et al., "Experimental evaluation of a new morphological approximation of the articular surfaces of the ankle joint," Journal of Biomechanics, vol. 53, no. 15, pp. 97-104, 2017.
[21] C. Belvedere, S. Siegler, A. Ensini et al., "Experimental evaluation of current and novel approximations of articular surfaces of the ankle joint," Journal of Biomechanics, vol. 75, no. 21, pp. 159-163, 2018.

[22] S. Nozaki, K. Watanabe, and M. Katayose, "Three-dimensional analysis of talar trochlea morphology: implications for subject-specific kinematics of the talocrural joint," Clinical Anatomy, vol. 29, no. 8, pp. 1066-1074, 2016.

[23] C. H. Barnett and J. R. Napier, "The axis of rotation at the ankle joint in man; its influence upon the form of the talus and the mobility of the fibula," Journal of Anatomy, vol. 86, no. 1, pp. 1-9, 1952.

[24] M. Wiewiorski, S. Hoechel, A. E. Anderson et al., "Computed tomographic evaluation of joint geometry in patients with end-stage ankle osteoarthritis," Foot \& Ankle International, vol. 37, no. 6, pp. 644-651, 2016.

[25] M. Wiewiorski, S. Hoechel, K. Wishart et al., "Computer tomographic evaluation of talar edge configuration for osteochondral graft transplantation," Clinical Anatomy, vol. 25, no. 6, pp. 773-780, 2012.

[26] C.-C. Kuo, H.-L. Lu, A. Leardini, T.-W. Lu, M.-Y. Kuo, and H.-C. Hsu, "Three-dimensional computer graphics-based ankle morphometry with computerized tomography for total ankle replacement design and positioning," Clinical Anatomy, vol. 27, no. 4, pp. 659-668, 2014.

[27] K. Islam, A. Dobbe, A. Komeili et al., "Symmetry analysis of talus bone," Bone \& Joint Research, vol. 3, no. 5, pp. 139-145, 2014.

[28] Y. Zhang, J. Xu, X. Wang et al., "An in vivo study of hindfoot $3 \mathrm{D}$ kinetics in stage II posterior tibial tendon dysfunction (PTTD) flatfoot based on weight-bearing CT scan," Bone \& Joint Research, vol. 2, no. 12, pp. 255-263, 2013.

[29] Y.-J. Zhang, J. Xu, Y. Wang, X.-J. Lin, and X. Ma, "Correlation between hindfoot joint three-dimensional kinematics and the changes of the medial arch angle in stage II posterior tibial tendon dysfunction flatfoot," Clinical Biomechanics, vol. 30, no. 2, pp. 153-158, 2015.

[30] K. Islam, A. Dobbe, K. Duke et al., "Three-dimensional geometric analysis of the talus for designing talar prosthetics," Proceedings of the Institution of Mechanical Engineers, Part H: Journal of Engineering in Medicine, vol. 228, no. 4, pp. 371-378, 2014.

[31] A. Trovato, M. El-Rich, S. Adeeb, S. Dhillon, and N. Jomha, "Geometric analysis of the talus and development of a generic talar prosthetic," Foot and Ankle Surgery, vol. 23, no. 2, pp. 89-94, 2017.

[32] Y. Tochigi, M. J. Rudert, C. L. Saltzman, A. Amendola, and T. D. Brown, "Contribution of articular surface geometry to ankle stabilization," The Journal of Bone and Joint SurgeryAmerican Volume, vol. 88, no. 12, pp. 2704-2713, 2006.

[33] O. Magerkurth, M. Knupp, H. Ledermann, and B. Hintermann, "Evaluation of hindfoot dimensions: a radiological study," Foot \& Ankle International, vol. 27, no. 8, pp. 612-616, 2006.

[34] A. Hayes, Y. Tochigi, and C. L. Saltzman, "Ankle morphometry on 3D-CT images," Iowa Orthopedic Journal, vol. 26, no. 26, pp. 1-4, 2006.

[35] D. H. Zhao, D. C. Huang, G. H. Zhang et al., "Gender variation in the shape of superior talar dome: a cadaver measurement based on Chinese population," BioMed Research International, vol. 2018, Article ID 6087871, 7 pages, 2018.

[36] S. P. Guy, M. A. Farndon, S. Sidhom, M. Al-Lami, C. Bennett, and N. J. London, "Gender differences in distal femoral morphology and the role of gender specific implants in total knee replacement: a prospective clinical study," The Knee, vol. 19, no. 1, pp. 28-31, 2012. 


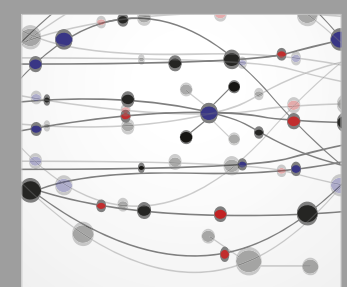

The Scientific World Journal
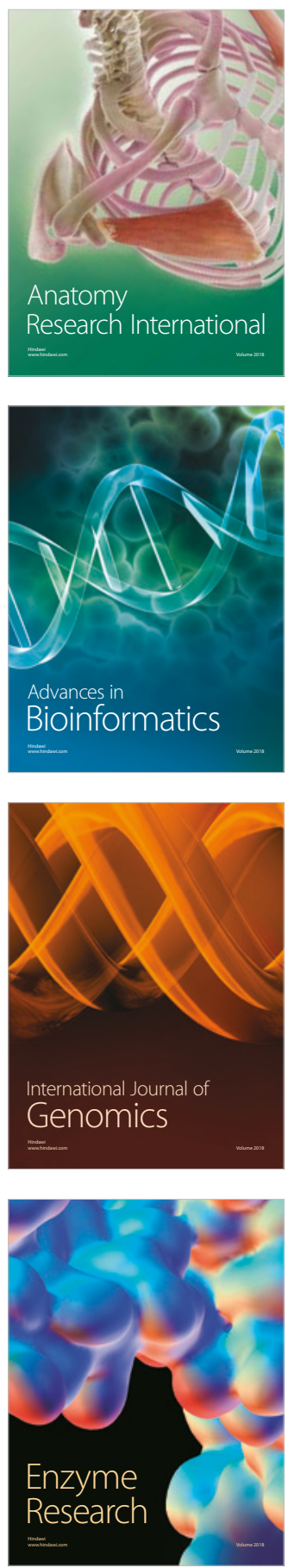
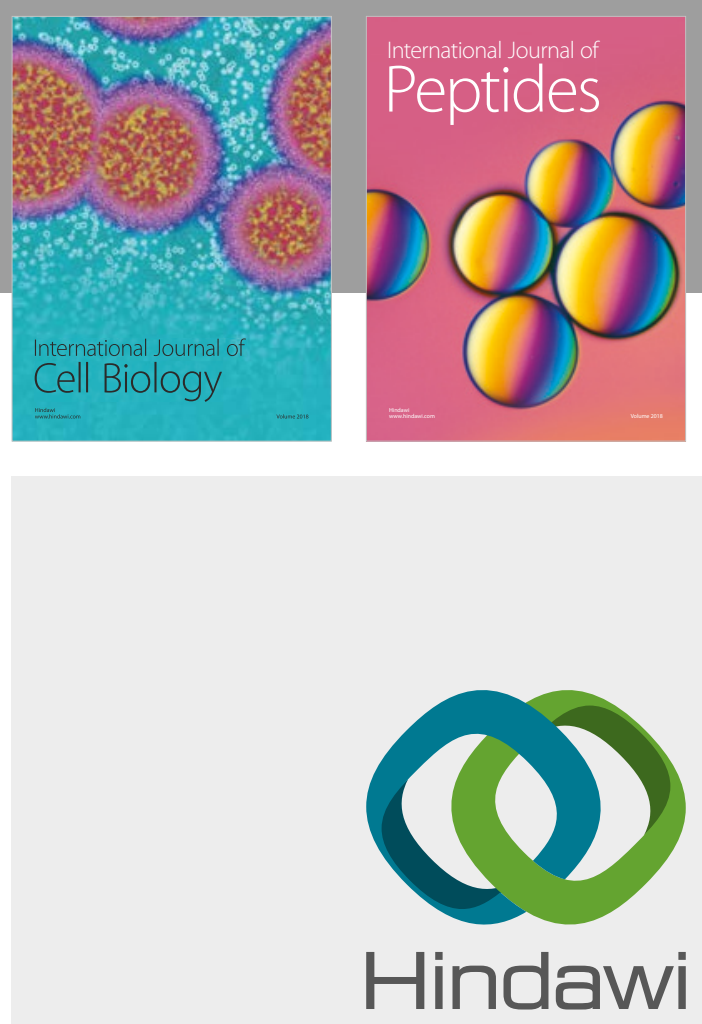

Submit your manuscripts at

www.hindawi.com
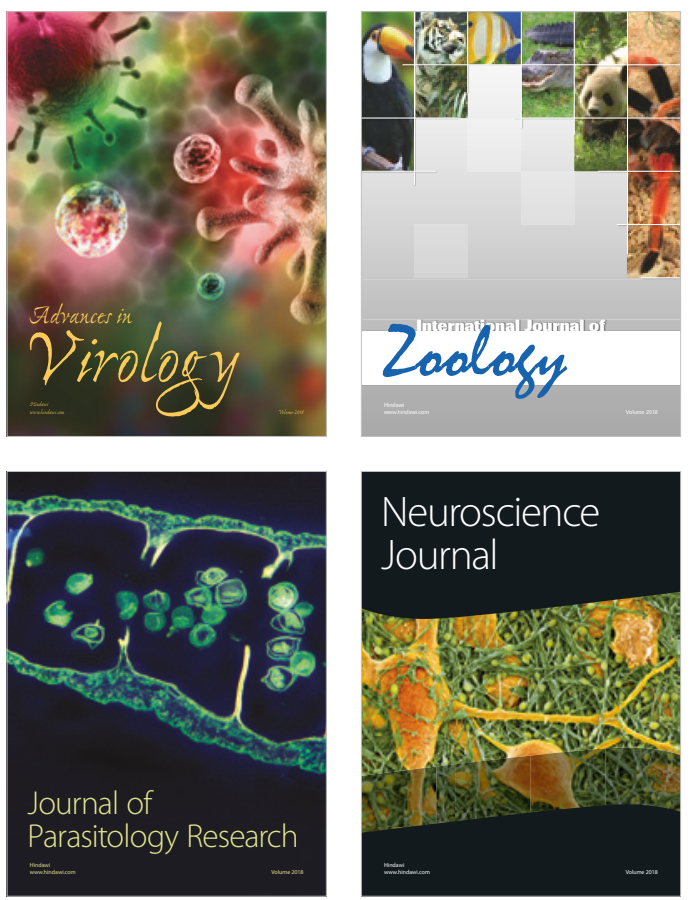
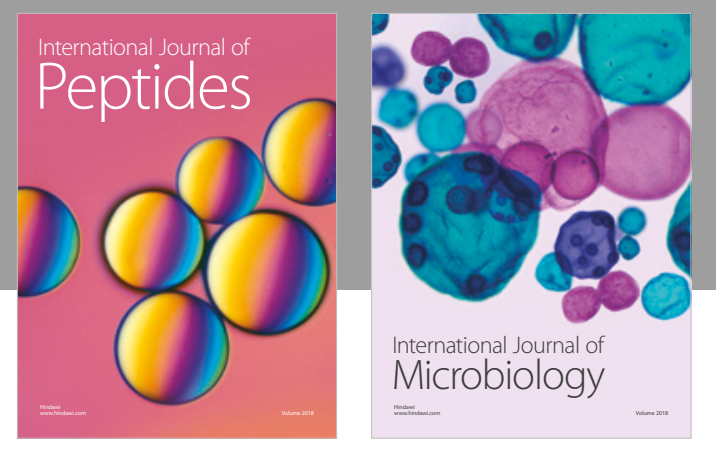

nternational Journal of Microbiology
Journal of
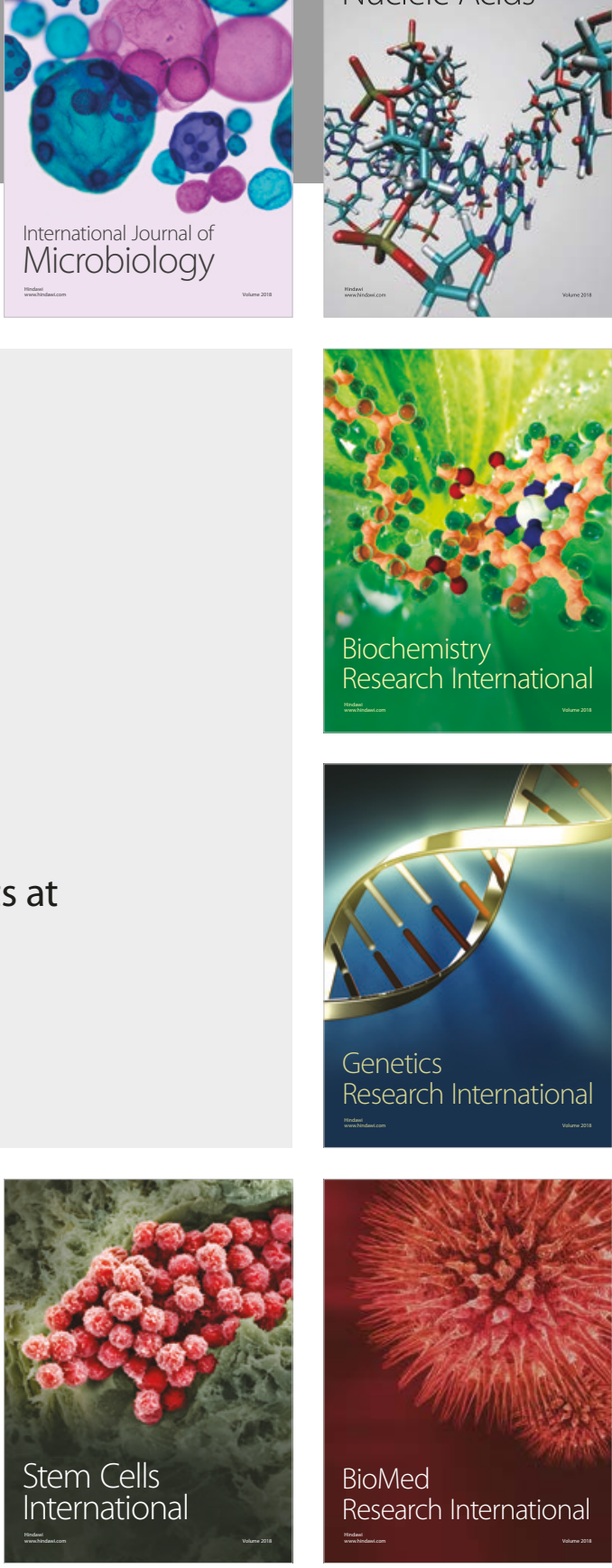
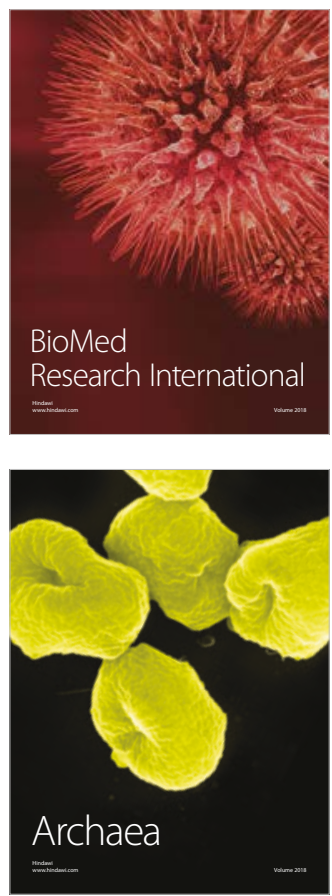